\title{
Ros3 (Lem3p/CDC50) Gene Dosage Is Implicated in Miltefosine Susceptibility in Leishmania (Viannia) braziliensis Clinical Isolates and in Leishmania (Leishmania) major
}

Caroline R. Espada,* Andreia Albuquerque-Wendt, Valentín Hornillos, Eva Gluenz, Adriano C. Coelho, and Silvia R. B. Uliana

Cite This: ACS Infect. Dis. 2021, 7, 849-858

Read Online

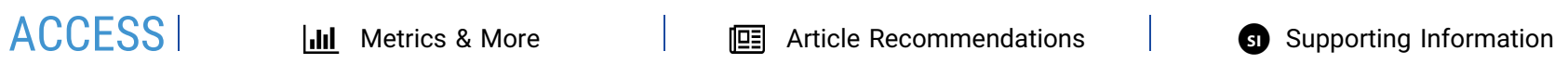

ABSTRACT: The Ros3 protein is a component of the MT-Ros3 transporter complex, considered as the main route of miltefosine entry in Leishmania. L. braziliensis clinical isolates presenting differences in miltefosine susceptibility and uptake were previously shown to differentially express ros3. In this work, we showed that the ros 3 gene copy number was increased in the isolate presenting the highest rates of miltefosine uptake and, thus, the highest

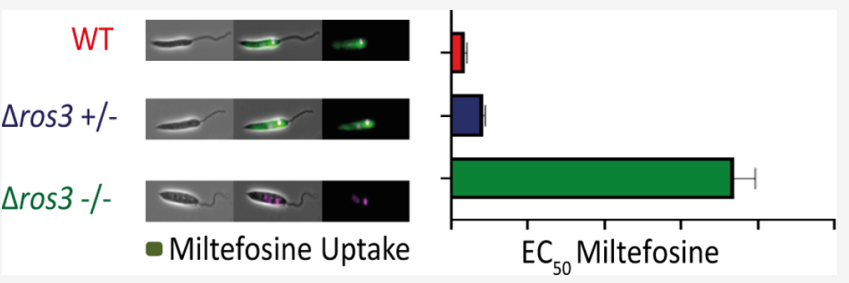
susceptibility to this drug. The role of the ros 3 gene dosage in miltefosine susceptibility was then investigated through a modulation of the gene copy number using two distinct approaches: through an overexpression of ros3 in a tolerant L. braziliensis clinical isolate and in L. major and by generating mono- and diallelic knockouts of this gene in L. major using clustered regularly interspaced short palindromic repeats (CRISPR) Cas9 (Cas = CRISPRassociated). Although the levels of ros 3 mRNA were increased at least 40 -fold in overexpressing clones, no significant reduction in the half-maximal effective concentration $\left(\mathrm{EC}_{50}\right)$ for miltefosine was observed in these parasites. The partial or complete deletion of ros 3 in L. major, in turn, resulted in a significant increase of 3 and 20 times, respectively, in the $\mathrm{EC}_{50}$ to miltefosine. We unequivocally showed that the ros 3 copy number is one of the factors involved in the differential susceptibility and uptake of miltefosine.

KEYWORDS: Leishmania braziliensis, miltefosine, drug resistance, clinical isolates, CRISPR/Cas9, treatment

Leishmania (Viannia) braziliensis is the main etiological agent of tegumentary leishmaniasis in Brazil. ${ }^{1,2}$ Infections caused by this species predominately manifest as localized cutaneous lesions but can also cause a severe mucosal disease or disseminated cutaneous manifestations. ${ }^{3}$ Therefore, a systemic treatment in these cases is mandatory, and the limitations of the treatment options in use in Brazil become even more alarming. ${ }^{4}$ The current therapy for leishmaniasis in Brazil relies on pentavalent antimonial and amphotericin B, both of them highly toxic and parenterally administered. ${ }^{5}$ Moreover, in some regions of Brazil the cure rates for cutaneous leishmaniasis (CL) upon treatment with meglumine antimoniate have drastically dropped to $\sim 50 \%$. ${ }^{6,7}$ For all these reasons, alternative therapies are highly needed in order to overcome these limitations.

Miltefosine (MF) is currently the most effective oral drug available for leishmaniasis treatment. ${ }^{8}$ In Brazil, recommendations for the use of MF for CL treatment were issued in 2018, but the drug is still not available for clinical use. ${ }^{9}$ Two clinical trials employing MF for CL treatment in patients infected with the Leishmania (Viannia) species of two different regions of Brazil showed cure rates of $\sim 70 \%{ }^{6,10}$ Although MF is not devoid of side effects, those are mostly milder than the side effects of antimony and amphotericin. ${ }^{11}$ Being the only oral drug in clinical use, MF is of great importance for leishmaniasis chemotherapy. However, the significant drop in cure rates observed in visceral leishmaniasis patients in India, together with the recent isolation of resistant parasites from patients previously treated with $\mathrm{MF}$, raised an alarm on the possible loss of this drug due to the selection of resistance. ${ }^{12-16}$ Moreover, recently data characterizing the in vitro susceptibility of L. infantum Brazilian clinical isolates recovered from patients enrolled in a clinical trial with MF for VL treatment revealed an alarming correlation between treatment failure and the intrinsic parasite susceptibility to MF. ${ }^{17}$

Miltefosine's entry into the Leishmania parasites relies on a P4-ATPase membrane transporter called miltefosine transporter (MT), which has as its main function the transport of phospholipids from the extracellular environment through the cell membrane. ${ }^{18}$ It is well-known that in vitro MF-selected parasites present a significant reduction in drug accumulation due to mutations in the $M T$ gene, which leads to a defective

Received: December 7, 2020

Published: March 16, 2021 
Table 1. Susceptibility to MF and ros3 Transcript Abundance in L. braziliensis Clinical Isolates

\begin{tabular}{|c|c|c|c|c|c|}
\hline \multicolumn{6}{|c|}{$\mathrm{EC}_{50} \pm \operatorname{SEM}^{a}(\mu \mathrm{M})$} \\
\hline abbreviation $^{b}$ & identification code ${ }^{c}$ & promastigotes & amastigotes & FC RNaseq $^{d}$ ( $p$-value) & FC real-time ${ }^{e}$ ( $p$-value) \\
\hline RS & MHOM/BR/1975/M2903 & $53.5 \pm 6.6$ & $2.7 \pm 0.2$ & ND & $-1.85(0.0993)$ \\
\hline$S$ & MHOM/BR/2005/LTCP16012 & $22.9 \pm 3.7$ & $0.8 \pm 0.1$ & 1.0 & 1.0 \\
\hline $\mathrm{T} 1$ & MHOM/BR/2006/LTCP 16907 & $101.2 \pm 6.0$ & $3.3 \pm 0.4$ & $-2.04(0.0069)$ & $-3.97(0.0198)$ \\
\hline $\mathrm{T} 2$ & MHOM/BR/2009/LTCP 19446 & $90.4 \pm 5.2$ & $4.2 \pm 0.2$ & $-2.10(0.0146)$ & $-3.93(0.0114)$ \\
\hline
\end{tabular}

${ }^{a} \mathrm{EC}_{50} \pm \mathrm{SEM}$ of MF for promastigotes and amastigotes of L. braziliensis clinical isolates and M2903 reference strain. Data previously published in Espada et al. ${ }^{26}{ }^{b}$ Code used for strain and isolates used in this study. ${ }^{c}$ International code of each isolate. ${ }^{d}$ Fold-change (FC) in ros 3 transcript abundance in tolerant isolates relative to the abundance in the sensitive isolate and adjusted $p$-value evaluated by three biological replicates of each isolate and M2903 reference strain. (ND) Not differentially expressed in this transcriptome. Data previously published in Espada et al. ${ }^{21}$ ${ }^{e}$ Normalized expression of ros 3 and adjusted $p$-value relative to $S$ isolate assessed by quantitative real-time RT-PCR. Data previously described in Espada et al. ${ }^{21}$

MF transport machinery. ${ }^{19,20}$ However, this reduced drug uptake was also shown to be present in parasites naturally less susceptible to MF without significant differences in the MT gene sequence and/or expression of this transporter. ${ }^{21,22}$

Another protein plays a key role in MF transport, the MT's beta subunit Ros3, which belongs to the Lem3p/CDC50 family. ${ }^{23}$ Together, they form the MT-Ros 3 complex, and both of them are indispensable for the complex functionality. ${ }^{24}$ The Ros3 subunit has been shown to play a key role in phospholipid transport and susceptibility of yeast to MF and edelfosine. $^{25}$ In Leishmania parasites, it has already been demonstrated that the absence or defects in Ros3 cause the retention of the whole MT-Ros3 complex in the endoplasmic reticulum and consequently resistance to $\mathrm{MF}^{24}$ Moreover, polymorphisms in both MT and ros 3 genes were described as responsible for the reduced susceptibility to MF in an $L$. infantum clinical isolate. ${ }^{15}$

We previously reported that L. braziliensis clinical isolates from Brazilian patients exhibited differences in susceptibility to $\mathrm{MF}^{26}$ as a result of differences in drug uptake. ${ }^{21}$ The levels of ros 3 mRNA were found to be decreased in tolerant isolates compared to a sensitive one, suggesting that a low abundance of this component of the MF transport complex could be the cause of a reduced susceptibility observed in tolerant isolates. ${ }^{21}$

Since gene and/or chromosome copy number variations have been implicated in the mechanisms of drug resistance and variation of susceptibility to drugs in Leishmania, ${ }^{27,28}$ we hypothesized that the differences in MF susceptibility, drug uptake, and ros 3 transcript abundance in these isolates might be the result of a differential ros 3 gene dosage in these parasites. Thus, in this work we investigated the copy number of the ros 3 gene in these L. braziliensis clinical isolates and the consequences of a differential ros 3 gene dosage for $\mathrm{MF}$ susceptibility by overexpressing and knocking out this gene in Leishmania.

\section{RESULTS}

Differential ros3 DNA Abundance among L. braziliensis Clinical Isolates. It was previously reported by our group that L. braziliensis clinical isolates obtained in Brazil presented variable susceptibility to $\mathrm{MF}^{26}$ Analysis of these isolates through transcriptome sequencing and quantitative real-time reverse transcription polymerase chain reaction (RTPCR) allowed the demonstration that the ros 3 mRNA abundance was upregulated in the most susceptible isolate (S) when compared to the M2903 reference strain (RS) and the two more tolerant isolates $(\mathrm{T} 1 \text { and } \mathrm{T} 2)^{21}$ (Table 1$)$.
Since the regulation of gene expression in trypanosomatids does not generally occur at a transcriptional level, a differential expression is mostly a result of differences in gene copy number or post-transcriptional regulation. ${ }^{28,29}$ Copy number variation $(\mathrm{CNV})$ was assessed in these isolates by a quantification of the ros3 DNA abundance by real-time PCR. Two housekeeping genes, gapdh and $t b p$, were chosen for normalization in these experiments after they showed a consistent expression between these isolates in the RNaseq data (data not shown). ${ }^{21}$

Significant differences in the ros3 DNA abundance were observed between $S$ and T1/T2 isolates using both gapdh and tbp as normalizers (Figure 1). The most susceptible isolate (S)

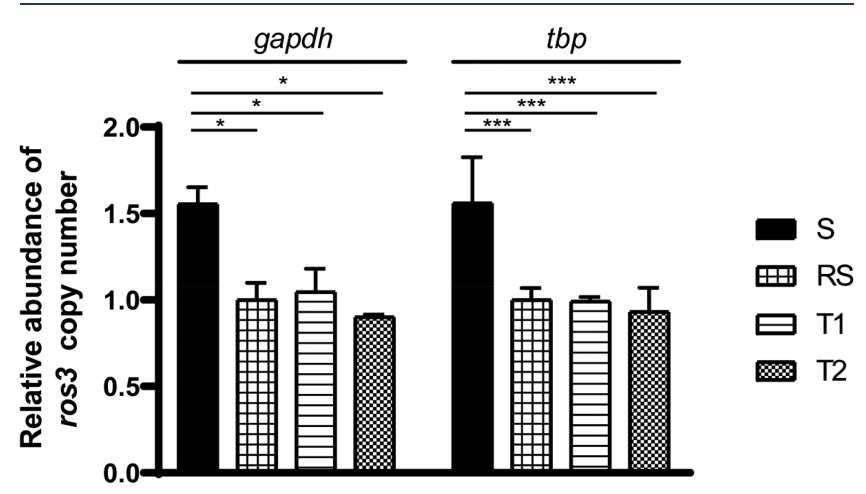

Figure 1. Relative abundance of ros3 DNA in L. braziliensis clinical isolates and reference strain (RS). Each bar represents the mean ros3 DNA molecules number in each isolate relative to the molecule numbers in RS. Relative abundance was assessed by real-time PCR in two independent experiments using two different normalizer genes (gapdh and $t b p$ ). Three independent biological replicates and three technical replicates were employed in each experiment. Statistical significance was determined using One-way ANOVA and Tukey's multiple comparison test. $(*) p<0.05$ and $(* *) p<0.001$.

presented a significant 0.5 -fold increased abundance of ros 3 DNA compared to the tolerant isolates in experiments employing different normalizer genes. The abundance profile of ros3 DNA molecules correlated with the abundance of transcripts of this gene previously reported by Espada et al. ${ }^{21}$ and is highlighted in Table 1. Interestingly, the DNA quantification did not reveal differences between the reference and $\mathrm{T}$ isolates, in spite of the previously noted changes in halfmaximal effective concentration $\left(\mathrm{EC}_{50}\right)$ and mRNA abundance.

Overexpressing ros3 in the Tolerant Isolate Does Not Increase MF Susceptibility. The increased abundance of 
ros3 DNA in the isolate $\mathrm{S}$ led us to investigate whether the addition of more copies of this gene in tolerant parasites would lead to an increase in susceptibility to MF. To test this hypothesis, we overexpressed the ros 3 gene in the isolate T2, which presented the highest $\mathrm{EC}_{50}$ to $\mathrm{MF}$. The ros 3 coding sequence was amplified from the $\mathrm{T} 2$ genome, cloned downstream to an L. tarentolae adenine phosphoribosyl (aptr) and upstream to a L. tarentolae calmodulin (camCB) untranslated region (UTR). The generated SR construct was then linearized and delivered by electroporation for integration in the SSU locus of this isolate. Recipient parasites were the T2 isolate and L. major FV-1 (Lm), included to verify if these findings would be similar for another Leishmania species. After a selection with hygromycin B in solid M199 seven clones of $\mathrm{T} 2$ and eight clones of $\mathrm{Lm}$ were screened by PCR for the presence and correct integration of the SR insert (Figure S1).

Three random T2 and three Lm SR clones presenting the SR cassette integrated in the right orientation were selected for an ros 3 mRNA abundance quantification. The relative abundance of the ros 3 mRNA in each clone relative to the WT parasites was assessed through real-time qPCR using tbp as a normalizing gene for $\mathrm{T} 2$ and $\mathrm{Lm}$ lines (Figure 2). An
A

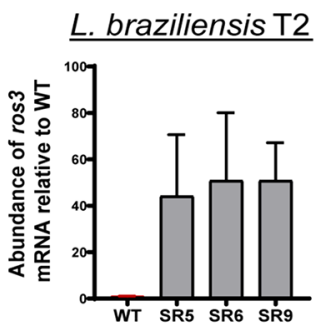

C

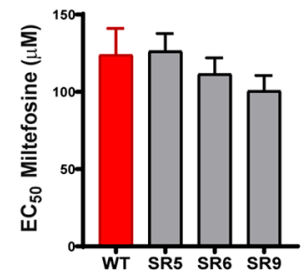

B

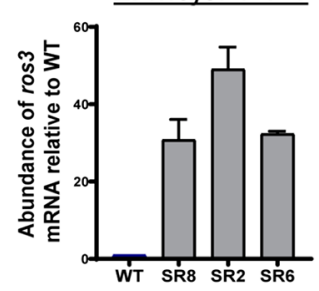

D

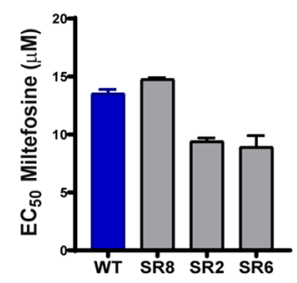

Figure 2. Relative abundance of the ros3 gene mRNA and phenotypic characterization in SR clones. Abundance of the ros 3 mRNA was quantified in each SR clone and WT parasites of L. braziliensis T2 (A) and L. major FV-1 (B) by real-time qPCR. Normalization was done using tbp gene $\mathrm{Ct}$ values, and each bar represents the mean \pm SEM abundance of the ros 3 transcript relative to WT parasites obtained in two independent experiments using two biological and three technical replicates (A, B). Susceptibility to MF was evaluated in SR clones and WT parasites of L. braziliensis T2 (C) and L. major FV-1 (D) by an MTT assay. Each bar represents the mean \pm SEM $\mathrm{EC}_{50}$ to MF obtained in three independent experiments performed in triplicate.

increase in transcript abundance was observed in both species. In L. braziliensis T2 SR clones, levels of ros 3 mRNA were increased 44- to 50-fold when compared to WT parasites (Figure 2A). In L. major SR clones, the increase in ros 3 mRNA levels varied from 30.8- to 49.0-fold compared to the WT parasite (Figure 2B).

The effects of ros 3 overexpression were investigated by an evaluation of log-phase promastigotes MF susceptibility in SR clones of $L$. braziliensis by 3-[4,5-dimethyl-2-thiazolyl]-2,5diphenyl-2H-tetrazolium bromide) (MTT). Compared to the $\mathrm{EC}_{50}$ determined for Lb WT $(123.9 \pm 17.08 \mu \mathrm{M})$, a nonstatistically significant decrease of $18 \%$ was observed for the clone SR9 $\left(\mathrm{EC}_{50}=100.6 \pm 9.94 \mu \mathrm{M}\right)$ (Figure $2 \mathrm{C}$ ).

In $\mathrm{Lm} \mathrm{SR}$ clones the $\mathrm{EC}_{50}$ reduction was more pronounced and significant for two out of three clones. A 30\% decrease in $\mathrm{EC}_{50}$ values was observed for clone SR8 $(8.93 \pm 0.97 \mu \mathrm{M})$ as compared to the $\mathrm{EC}_{50}$ calculated for the WT parasite (13.54 \pm $0.34 \mu \mathrm{M}$ ) (Figure 2D). However, no significant correlation between the ros 3 mRNA abundance and MF susceptibility was found for either L. braziliensis or L. major ros3 overexpressor clones $(r=-0.800$ and $p=0.333 ; r=-0.600$ and $p=0.4167$, Spearman's correlation test, respectively).

Increasing the hygromycin selection pressure from 32 to 128 $\mu \mathrm{g} / \mathrm{mL}$ did not lead to a more pronounced reduction in $\mathrm{EC}_{50}$ values in either species. In T2 SR clones, the $\mathrm{EC}_{50}$ of SR2 and SR5 decreased from 111.53 and $126.64 \mu \mathrm{M}$ to 102.3 and 119.5 $\mu \mathrm{M}$, respectively. In Lm SR clones the $\mathrm{EC}_{50}$ of SR2 and SR8 under increased hygromycin B pressure changed from 10.8 and 14.7 to 13.5 and $16.5 \mu \mathrm{M}$, respectively.

Partial or Complete Removal of ros3 Reduces Susceptibility and Uptake of MF in L. major. As an alternative strategy to evaluate the role of the ros3 gene dosage effect on MF susceptibility, an Lm strain constitutively expressing Cas9 and T7 RNA polymerase (Lm Cas9/T7) was used to generate mono (partial) and diallelic (complete) knockouts for ros3 using CRISPR/Cas9.

For that, small guide RNAs (sgRNAs) coding template and donor DNAs were generated by PCR in vitro and delivered to the parasite, driving the Cas9-mediated break and incorporation of the donor DNA containing a blasticidin resistance (BlastR) gene by homologous recombination, replacing the complete ros3 open read frame (ORF).

After the selection with blasticidin, single and complete knockouts (KOs) for the ros 3 gene were confirmed by PCR in the heterogeneous parasite population that was previously cloned by serial dilution (Figure 3 ). To verify the presence or absence of ros 3 in the clones recovered from ros $3 \mathrm{KO}$ experiments, primers annealing outside the ORF were used. The presence of the ros 3 gene was identified by the presence of a $1.4 \mathrm{~kb}$ fragment, whereas its substitution by a Blast-R gene

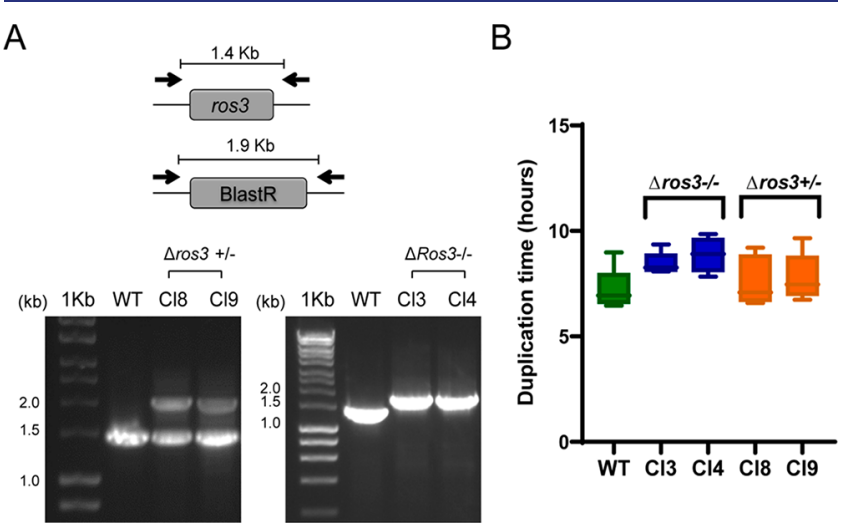

Figure 3. ros3 knockout verification in recovered clones. (A) Schematic representation of the strategy used for ORF KO verification in blasticidin-resistant clones. In diallelic knockouts, Blast- $\mathrm{R}$ was amplified and resulted in a $1.9 \mathrm{~kb}$ fragment, whereas in monoallelic ros $3 \mathrm{KOs}$ both Blast-R and ros 3 are amplified resulting in two bands of 1.9 and $1.4 \mathrm{~kb}$, respectively. (B) Parasite doubling time for each incomplete $(\Delta \operatorname{ros} 3+/- \pm)$ and complete $(\Delta \operatorname{ros} 3-/-) \mathrm{KO}$ clones and for WT L. major Cas9/T7. Each box represents the mean \pm SEM of the duplication time evaluated during $4 \mathrm{~d}$. 
A

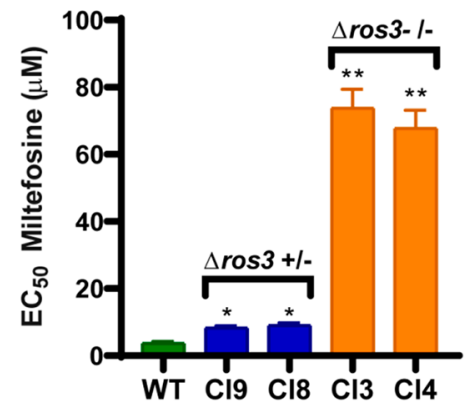

B

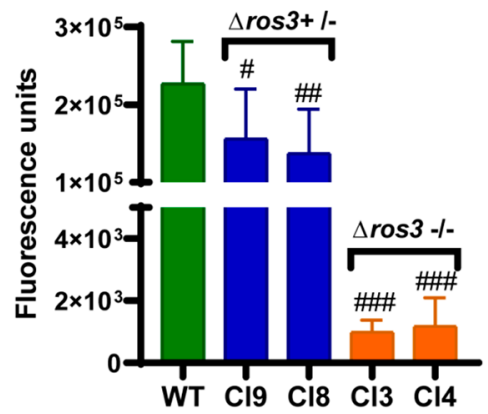

C

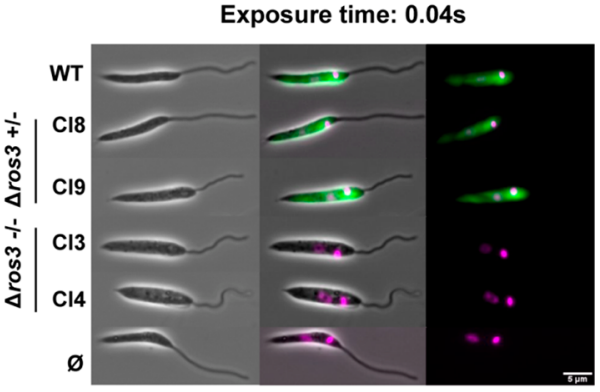

Exposure time: $4.0 \mathrm{~s}$

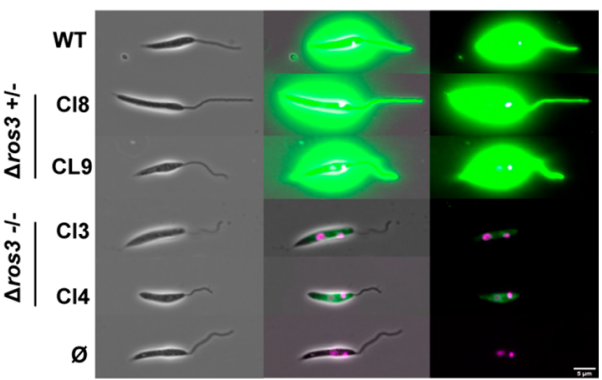

Figure 4. Susceptibility and uptake of MF in L. major ros3 mono- and diallelic KOs. (A) The $\mathrm{EC}_{50}$ of $\mathrm{MF}$ was determined for the L. major Cas9/T7 strain and for mono- and diallelic knockouts by MTT. Mean and SEM results of three or more independent experiments were calculated. (*) $p<$ 0.005. (**) $p$ 0.0001. (B) Uptake of MT-EtBDP in monoallelic (Cl8 and Cl9) and diallelic ( $\mathrm{Cl} 3$ and $\mathrm{Cl} 4)$ ros3 knockouts. (\#) $p<0.01$; (\#\#) $p<$ 0.001; (\#\#\#) $p<0.0001$. Uptake of MT-EtBDPY was evaluated by flow cytometry. A reduction in fluorescence (FLH-1) inside parasites was observed in ros3 incomplete knockouts. In complete knockouts, the reduction was even higher. (C) Evaluation of MT-EtBDP uptake in WT (Lm Cas9/T7) and in ros3 monoallelic ( $\mathrm{Cl} 8$ and $\mathrm{Cl}$ ) and diallelic ( $\mathrm{Cl} 3$ and $\mathrm{Cl} 4$ ) knockouts by fluorescence microscopy. Different exposure times of 0.04 and $4 \mathrm{~s}$ were used to confirm the presence of the labeled MF inside parasites. As a background, the control WT Cas $9 / \mathrm{T} 7$ incubated without MT-EtBDPY was exposed to the same conditions for image acquisition.

resulted in the amplification of a $1.9 \mathrm{~kb}$ fragment (Figure 3A). Six clones with incomplete and six clones with complete deletions of ros3 (Figure 3) were identified. Two incomplete ( $\mathrm{Cl} 8$ and $\mathrm{Cl} 9)$ and two complete $(\mathrm{Cl} 3$ and $\mathrm{Cl} 4)$ ros3-deficient mutants were selected for MF susceptibility and uptake characterizations.

To exclude the possible interference of growth rates between the mutants in the viability assays, the doubling time of the generated lines in parallel with the WT parasites was determined as described in the Methods Section. Although a mild increase in the doubling time was observed in complete knockouts ( $\mathrm{Cl} 3$ and $\mathrm{Cl} 4$ ), this difference was not significant [analysis of variance (ANOVA) and Tukey's multiple comparisons test] (Figure 3). Other features including size, shape, or motility were visually inspected under a bright fieldinverted microscope. None of the clonal mutant population cultures presented differences detectable to the human eye (Figure 4C).

The susceptibility of partial and complete knockouts to MF was determined by MTT. Initially, the susceptibility of L. major Cas9/T7 was compared to the L. major WT line (the background in which L. major Cas9/T7 was generated) in order to evaluate if the modified parasite could behave differently regarding MF susceptibility. No significant differences were observed between $\mathrm{Lm}$ WT $\left(\mathrm{EC}_{50}=6.57 \pm 0.89\right.$ $\mu \mathrm{M})$ and the Lm WT Cas9/T7 strain $\left(\mathrm{EC}_{50}=3.46 \pm 0.48\right.$ $\mu \mathrm{M})$ (non-parametric t-test).

When compared to Lm Cas9/T7 WT parasites, a significant approximately threefold reduction $(p>0.002)$ in $\mathrm{EC}_{50}$ values for MF was observed for monoallelic deleted ros 3 mutants
(Table 2). When the two alleles of ros 3 were removed (diallelic deleted ros 3 mutants), a more pronounced reduction in the

Table 2. Susceptibility of L. major ros3 Nono- and Diallelic Knockouts to MF

\begin{tabular}{lcc}
\multicolumn{1}{c}{ sample } & $\mathrm{EC}_{50} \pm \mathrm{SEM}^{a}(\mu \mathrm{M})$ & activity index $^{b}(\mathrm{AI})$ \\
$\mathrm{Lm} \mathrm{WT}$ & $6.57 \pm 0.89$ & \\
$\mathrm{Lm} \mathrm{WT} \mathrm{Cas9/T7}$ & $3.46 \pm 0.48$ & \\
$\Delta \operatorname{ros} 3 \pm \mathrm{Cl} 8$ & $10.22 \pm 1.27$ & 2.95 \\
$\Delta \operatorname{ros} 3 \pm \mathrm{Cl} 9$ & $9.66 \pm 1.43$ & 2.79 \\
$\Delta \operatorname{ros} 3-/-\mathrm{Cl} 3$ & $73.82 \pm 5.53$ & 21.33 \\
$\Delta \operatorname{ros} 3-/-\mathrm{Cl} 4$ & $71.47 \pm 5.49$ & 20.65
\end{tabular}

${ }^{a} \mathrm{EC}_{50} \pm \mathrm{SEM}$ of MF for promastigotes determined by MTT assay. ${ }^{b}$ Activity index (AI) was calculated by dividing the $\mathrm{EC}_{50}$ of each clone by the $\mathrm{EC}_{50}$ of the reference parasite L. major Friedlin Cas9/T7.

susceptibility to MF ( $\sim 20$-fold reduction) was detected ( $p<$ 0.0001) (Table 2 and Figure 4A).

The uptake of MF in mono- and diallelic knockouts was determined by flow cytometry using MF labeled with 11$\left(4^{\prime}, 4^{\prime}\right.$-difluoro-6' -ethy $\left[11-\left(4^{\prime}, 4^{\prime}\right.\right.$-difluoro-6' -ethyl-1', $3^{\prime}, 5^{\prime}, 7^{\prime}$ tetramethyl-4' -bora-3' a, $4^{\prime}$ a-diaza-s-indacen-2' -yl)-undecylphosphocholine] (MT-EtBDPY) and compared to Lm Cas9/ T7 WT. In the absence of one allele of ros3, the amount of fluorescence inside the parasite presented a mild but significant reduction. However, in ros 3 diallelic knockouts, the uptake is reduced 100-fold compared to that of WT Cas9/T7. These data suggested that, in the absence of one or two copies of ros3, the MF entry in $L$ major is impaired (Figure $4 \mathrm{~B}$ ). 
The drug uptake variation was also demonstrated by fluorescence microscopy (Figure 4C). With $0.04 \mathrm{~s}$ of exposure, no fluorescence was observed inside Ros 3 null mutants, while positive labeling was seen in WT parasites and incomplete knockouts. However, after longer exposures (4s), a weak MF fluorescence signal was observed in complete knockouts, demonstrating that some MF uptake happened even in the complete absence of ros3 (Figure 4C).

\section{DISCUSSION}

The susceptibility to MF was found to be variable among $L$. braziliensis clinical isolates, raising the concern of intrinsic tolerance in isolates circulating in Brazil. ${ }^{26} \mathrm{~A}$ further investigation of the mechanisms behind the differential susceptibility to MF in these isolates revealed differences in drug uptake and in the abundance of the ros 3 transcript, an essential component of the MF transport machinery. ${ }^{21,24}$ Considering the nature of gene expression regulation in Leishmania, ${ }^{30}$ the high genome plasticity, ${ }^{31,32}$ and the previous association of gene copy number variation ( $\mathrm{CNV}$ ) with drug resistance in these organisms, ${ }^{33}$ in this work we investigated whether or not a differential ros 3 mRNA abundance in $L$. braziliensis clinical isolates was related to variability in ros3 gene dosage. Furthermore, using different DNA manipulation approaches we evaluated if susceptibility to MF could be modulated by the addition or removal of ros 3 gene copies, mimicking a $\mathrm{CNV}$ condition.

A 0.5 -fold increase in the ros3 DNA abundance was found in the $L$. braziliensis susceptible isolate when compared to tolerant isolates and the reference strain. This indicated the presence of an extra copy of ros3, the most likely reason for the increased abundance of ros 3 mRNA observed. The ros 3 extra copy may represent an isolated event of gene duplication or a chromosome 32 tetrasomy, but further investigation about the chromosome content in these isolates has not been performed yet.

These findings then led us to investigate whether an alteration in the ros 3 gene dosage was enough to modulate the susceptibility to MF. On the one hand, the integration of an extra ros3 copy in the genome significantly increased the mRNA abundance in overexpressing clones. However, the accumulation of ros 3 transcripts did not lead to significant changes in the MF susceptibility in these parasites, suggesting that the increase of ros 3 transcripts alone was not capable of modulating the MF susceptibility in L. braziliensis T2 and $L$. major. On the other hand, the generation of mono- and diallelic ros3 knockouts using CRISPR/Cas9 in L.major led to 2 -fold and 20-fold increases in the $\mathrm{EC}_{50}$ to $\mathrm{MF}$, respectively.

The observation of the unchanged susceptibility to MF in $L$. braziliensis $\mathrm{T} 2$ and in L. major was surprising. The expression system employed for ros 3 overexpression herein (pLEXSY) is capable of inducing the expression of exogenous and endogenous genes in different Leishmania species, including L. braziliensis. ${ }^{34-37}$ Moreover, differential ros3 and $M T$ expressions have already been shown by others to play a role in the susceptibility of Leishmania to MF. ${ }^{22}$ An L. braziliensis Peruvian isolate and the Brazilian reference strain M2904 were shown to be 6-10-fold less susceptible to MF when compared to $L$. donovani due to a reduced expression of Ros 3 in the plasma membrane of L. braziliensis. ${ }^{22}$ This is the same range of variation in susceptibility to MF encountered among Brazilian L. braziliensis clinical isolates. ${ }^{26}$ Additionally, the same study showed that $L$. braziliensis overexpressing ros 3 demonstrated a 3.5-fold reduction in $\mathrm{MF} \mathrm{EC}_{50}$. Importantly, they have shown that, in this context of ros 3 overexpression in Leishmania, there is an increase not only in Ros3 protein abundance in plasma membrane but also of MT protein, suggesting that ros3 overexpression triggers an endogenous $M T$ overexpression, since the complete MT-Ros3 complex is essential for MF uptake. ${ }^{22}$ It is possible therefore that the susceptibility of Ros3 overexpressor mutants was unchanged because they lacked the necessary MT to compound the transporter complex MTRos3.

However, one important limitation in this study is the lack of a demonstration of an increased abundance of the Ros 3 protein in the overexpressing mutants. Various attempts of immunodetection and protein tagging did not produce clearcut results, so this remains to be achieved. Therefore, we must consider biological factors that could explain the lack of phenotype in the overexpressor mutants. An overexpression was achieved using the L. braziliensis T2 ros 3 coding sequence upstream to a heterologous UTR element. The lack of ros3 UTR elements may have hampered a proper mRNA processing and translation. ${ }^{38}$ Where overexpression in L. major is concerned, the limited identity (72\%) between L. braziliensis and L. major ros 3 coding sequences could potentially lead to interference in Ros3 folding, interaction with MT, and membrane insertion when expressed in L. major. ${ }^{23}$

Moreover, besides ros3, another 35 genes were shown to be differentially expressed between sensitive and tolerant isolates, ${ }^{21}$ and those could represent indispensable partners for an effective change in the MF uptake. Therefore, increasing the ros 3 transcript abundance through the methodology employed herein was not enough to sensitize $L$. braziliensis $\mathrm{T} 2$ and $L$. major to MF.

However, results obtained employing a loss of function approach revealed that the knockout of ros 3 modulates the susceptibility and uptake of MF in a gene-dosage-dependent way. The generation of complete and incomplete ros 3 knockout in L. major caused a significant decrease in uptake and in the susceptibility to MF, suggesting that the presence of this gene is critical for the susceptibility to MF. Similar results were observed in $L$. donovani, which presented a reduction in the susceptibility to MF of 1.7- and 14.2-fold in ros 3 monoand diallelic knockouts, respectively, suggesting that the MF tolerance phenotype caused by the reduction in ros 3 gene dosage is not a species-specific phenotype. Interestingly, these values are also comparable to the reduction of 1.9- and 13.7fold observed in the context of MT mono- and diallelic knockouts, which reinforces the codependence of both proteins. $^{24}$

In addition, the complete removal of ros 3 did not abolish MF internalization completely, as residual fluorescent MF was observed inside the diallelic knockouts, suggesting that other routes for the internalization of $\mathrm{MF}$, such as endocytosis or diffusion after incorporation into cell membranes, may be involved, even if poorly. ${ }^{39}$ If in the absence of the MT-Ros3 complex, diffusion through the membrane occurs in significant levels, variations in the composition and structure of plasma membrane in Leishmania parasites might also play a role in a differential susceptibility to MF.

To the best of our knowledge, this is the first demonstration of ros3 gene dosage described for Leishmania clinical isolates associated with a differential susceptibility and uptake of miltefosine. However, the dependence of the MT-Ros3 complex for MF transport has been repeatedly shown as the 
Achille's heel of MF efficacy in Leishmania parasites either by acquisition of inactivating mutations in these genes ${ }^{15,19,20,40,41}$ or by a differential expression of this complex in Leishmania plasma membrane. $^{22}$

Taken together, these results reinforced the role of the Ros3 subunit as a limiting factor for the MF uptake in Leishmania parasites and demonstrated for the first time that the ros 3 gene dosage plays a role in a differential susceptibility to MF not only in L. braziliensis isolates never exposed to MF but also in L. major.

The high cure rates, ${ }^{42,43}$ together with the high intracellular concentrations of MF achieved during therapy ${ }^{44,45}$ and the low number of cases of resistant parasites recovered after treatment with $\mathrm{MF},{ }^{13,15}$ are good indicatives that the variations observed in these L. braziliensis isolates are not enough to cause a treatment failure. However, it is important once again to highlight that MT-Ros3 is repeatedly being described as the cause of susceptibility reduction, not only in parasites selected in vitro under drug pressure but also in an isolate recovered after VL treatment with MF failure. ${ }^{15}$ In this scenario, our findings highly encourage the search for new drug therapy schemes, such as drug combinations that could enhance the MF activity, or even modifications in MF molecule that could promote the entrance of the drug by an alternative route in an attempt to avoid selection of resistant parasites and loss of the only effective oral drug for leishmaniasis treatment.

\section{CONCLUSIONS}

Being the only oral drug currently in use for leishmaniasis treatment, preventing a loss of MF due to resistance is a necessary effort. Our study reinforce the role of MT-Ros3 machinery in MF resistance by showing the direct effect of ros3 gene dosage in MF susceptibility and uptake not only in longterm laboratory-cultured Leishmania reference strains or parasites selected for resistance but also in L. braziliensis clinical isolates not previously exposed to MF. Our results encourage the search for new variants of MF molecule, different drug-delivery systems, or even coadministration with other molecules that could enhance MF transport and overcome the stringent dependence of active transport through the MT-Ros3 complex.

\section{METHODS}

Chemical Compounds. Miltefosine and MTT were purchased from Sigma-Aldrich and diluted in sterile water and phosphate-buffered solution (PBS), respectively. The BODIPY-labeled MF MT-EtBDP was kindly donated by Dr. A. U. Acuña (Instituto de Química-Física "Rocasolano", CSIC) and prepared as described. ${ }^{46}$ Hygromycin B and blasticidin S hydrochloride were purchased from Melford Laboratories Ltd.

Cultivation of Leishmania Parasites. The cell lines used in this work were: the parental strain of L. major Friedlin FV-1 (MHOM/IL/1980/Friedlin) (Lm) and the modified Cas9/ T7-expressing L. major Friedlin FV-1 (Lm Cas9/T7); ${ }^{47}$ the $L$. braziliensis reference strain (RS) (MHOM/BR/1975/M2903) and three L. braziliensis Brazilian clinical isolates, namely, MHOM/BR/2005/LTCP16012 (named S, for Sensitive), MHOM/BR/2006/LTCP16907 (T1, for Tolerant 1), and MHOM/BR/2009/LTCP19446 (T2, for Tolerant 2). The susceptibility of these isolates to MF was previously reported ${ }^{26}$ (Table 1).
Promastigotes of Leishmania were cultivated at $28{ }^{\circ} \mathrm{C}$ in M199 medium (Sigma-Aldrich) supplemented with $2.2 \mathrm{~g} / \mathrm{L}$ $\mathrm{NaHCO}_{3}, \quad 0.005 \%$ hemein, $40 \mathrm{mM}$ 4-(2-hydroxyethyl)piperazine-1-ethanesulfonic acid (HEPES), $\mathrm{pH} 7.4$, and 10\% heat-inactivated fetal calf serum (FCS). For L. braziliensis, 2\% male urine was added to the culture. Transfectants were maintained in MM199 media, which was made by diluting M199 media powder and supplementing with $2.2 \mathrm{~g} / \mathrm{L}$ $\mathrm{NaHCO}_{3}, 0.0025 \%$ hemein, $40 \mathrm{mM}$ HEPES, pH 7.4, 0.1 $\mathrm{mM}$ adenine hemisulfate, $1.2 \mu \mathrm{g} / \mathrm{mL}$ biopterin, and $20 \%$ FCS. The appropriate selection drug was added at $32 \mu \mathrm{g} \mathrm{mL}^{-1}$ hygromycin B or $5 \mu \mathrm{g} \mathrm{mL}^{-1}$ blasticidin $\mathrm{S}$ hydrochloride.

Generation of Parasites Overexpressing of ros3 Gene. For the ros 3 overexpression in the L. braziliensis T2 isolate and L. major Friedlin FV-1 strain, the open reading frame of the gene ros3 (LbrM.32.0580) was amplified from $L$. braziliensis T2 total DNA using the primer pair SR BglII-Fow and SR_NotI-Rev (Table S1) and cloned into the plasmid pLEXSY-hyg2 (Jena Biosciences). In this expression vector, the gene of interest is under the regulation of $L$. tarentolae adenine phosphoribosyl transferase (aprt) UTR at $5^{\prime}$ and calmodulin (camCB) UTR at $3^{\prime} .^{48}$ The generated construct (SR) was then linearized with the restriction enzyme SwaI, and purified cassette pLEXSY-hyg2-SR was delivered to L. braziliensis T2 isolate and L. major Friedlin FV-1 parasites for integration in the small subunit of rDNA (SSU) by electroporation, as previously described. ${ }^{49}$ Transgenic parasites overexpressing the ros3 gene (SR clones) were selected in a semisolid M199 medium supplemented with $32 \mu \mathrm{g} / \mathrm{mL}$ hygromycin $\mathrm{B}$ as described. ${ }^{50}$ Recovered clones were then maintained in liquid M199 in the presence of $32 \mu \mathrm{g} / \mathrm{mL}$ hygromycin B. Genomic DNA was extracted using the protocol described by Rotureau et al., ${ }^{51}$ and integration of the SR cassette into the SSU rDNA locus was confirmed by PCR using the primers provided with pLEXSY-hyg2 F3001, A264, F3002, and A384 (Jena Bioscience) (Table S1).

Quantitative Real-Time PCR. The abundance of ros3 mRNA and DNA in SR clones and isolates was quantified by real-time RT-PCR (qPCR) using total RNA or genomic DNA as templates, respectively. For the mRNA quantification, cDNA was synthesized from total RNA using MuLV Reverse Transcriptase (Applied Biosystems). Briefly, $6 \mu \mathrm{g}$ of DNasetreated RNA was incubated with $1 \mu \mathrm{g}$ of random primers (Thermo Fischer Scientific) for $10 \mathrm{~min}$ at $70{ }^{\circ} \mathrm{C}$. After this period 1X MulV-RT buffer, $0.01 \mathrm{mM}$ dithiothreitol (DTT), $5.5 \mathrm{mM} \mathrm{MgCl}_{2}$, and $1 \mathrm{mM}$ dNTPs were added to the system and incubated at $42{ }^{\circ} \mathrm{C}$ for $2 \mathrm{~min}$. Reverse Transcriptase was then added to the RT+ tubes but not the RT - tubes (control of DNA absence), and the reaction was incubated at $25^{\circ} \mathrm{C}$ for $10 \mathrm{~min}$, followed by incubations at $48{ }^{\circ} \mathrm{C}$ for $30 \mathrm{~min}$ and at 95 ${ }^{\circ} \mathrm{C}$ for $5 \mathrm{~min}$ according to the manufacturer's instructions.

One hundred nanograms of in vitro synthesized cDNA was used a as template for $\mathrm{qPCR}$, which was performed in a StepOne Plus System (Applied Bios Systems) using SYBR Green PCR Master Mix (Thermo Fisher Scientific). The following program was used: $95{ }^{\circ} \mathrm{C}$ for $10 \mathrm{~min}$ followed by 40 cycles at $95{ }^{\circ} \mathrm{C}$ for $15 \mathrm{~s}, 60^{\circ} \mathrm{C}$ for $60 \mathrm{~s}$, and $72{ }^{\circ} \mathrm{C}$ for $20 \mathrm{~s}$. The 163 base pair (bp) ros3 gene fragment was amplified using the primer pair LbLm_Ros3-F and LbLm_Ros3-R (Table S1). The housekeeping glyceraldehyde 3-phosphate dehydrogenase (gapdh) and tata-box-binding protein $(t b p)$ coding genes were used for normalization and amplified using the primer pair $g a p d h-\mathrm{F}$ and gapdh-R and Lb_tbp-F/Lm_tbp-F and 
LbLm_tbp-R, respectively (Table S1). Three biological replicates and three technical replicates of each sample were evaluated for ros3 and gapdh/tbp mRNA and DNA abundance determination. The threshold cycle $(\mathrm{Ct})$ obtained for ros 3 in each sample was normalized by the $\mathrm{Ct}$ of the gapdh/tbp genes. The $2^{-\Delta \Delta \mathrm{Ct}}$ equation was used to determine the expression of ros 3 genes relative to $\mathrm{RS}$, in the case of isolates, or to the wild type (WT) parasites when SR clones were characterized, respectively. ${ }^{52} 2^{-\Delta \Delta \mathrm{Ct}}$ values were then plotted on GraphPad Prism 6, and statistical analyses were performed using a oneway ANOVA analysis followed by Tukey's multiple comparison tests.

For quantifying the copy number of the ros3 gene, the samples were submitted to real-time PCR together with a standard curve using the pGEM-T-Ros3-M2903 plasmid (previously available in the laboratory). ${ }^{21} \mathrm{~A}$ linear regression of $\mathrm{Ct}$ values and ros 3 molecule number was constructed based on the data obtained for the standard curve and employed to determine the number of molecules in each sample. After normalization by $t b p$ or gapdh molecule values, the relative abundance of ros 3 was calculated by dividing the normalized amount of ros3 in each isolate by the amount in RS.

Generation of Mono- and Diallelic Knockouts for the ros3 Gene. Knockout mutants of this study were generated by CRISPR/Cas9 technology and the LeishGEdit toolkit on the background of L. major overexpressing Cas9/T7. ${ }^{53}$ Primer sequences for the PCR generation of sgRNA templates and donor DNAs for the ros3 gene ID (LmjF.32.0510) were selected using LeishGEdit (http://www.leishgedit.net/). ${ }^{53}$

The sgRNA templates for target gene cleavage were generated by PCR reactions using the G00 primer together with the 5' (LmRos3 5'sgRNA) or 3'sgRNA (LmRos3_3'sgRNA) LeishGEdit primers in individual tubes (Table S1). Donor DNA for generation of ros3 knockouts was also obtained by PCR reactions using the pTBlast_v1 plasmid as a DNA template and Upstream Forward Primer (LmRos3_UFP) and Downstream Reverse Primer (LmRos3_DRP) initiators (Table S1). Detailed protocols used for PCR reactions are described in Beneke and Gluenz. ${ }^{53}$

The delivery of sgRNA templates and donor DNA to $1 \times$ $10^{7} \mathrm{Lm}$ Cas9/T7 log-phase promastigotes was done by a transfection with Amaxa Nucleofector using program X-001 in a transfection buffer as previously described. ${ }^{53}$ After transfections, parasites were added to flasks containing MM199, and after $6 \mathrm{~h}, 5 \mu \mathrm{g} / \mathrm{mL}$ blasticidin was added to the culture. ${ }^{53}$ After two splits in 1:100 proportion, the recovered population was cloned in 96-well plates in three different proportions 0.1, 1.0, and 10 promastigotes $/ \mathrm{ml}$. Population was considered to be clonal when no more than $30 \%$ of the wells in each dilution presented growth.

Monoallelic (single) and diallelic (double) knockouts were verified through PCR reactions using primers LmRos3_UTR-F and LmRos3_UTR-R, which anneal outside the ros3 ORF. The presence of WT ros 3 results in the amplification of a 1.4 $\mathrm{kb}$ fragment, whereas the substitution by the Blast- $\mathrm{R}$ gene would result in the amplification of a $1.9 \mathrm{~kb}$ fragment. DNA obtained from the ros 3 transfectant population and of $\mathrm{Lm}$ Cas9/T7 were used as positive controls for partial $\mathrm{KO}$ and target gene presence, respectively.

Doubling Time Measurement. For doubling time characterization in mutants and WT parasites, the culture density was adjusted to $1 \times 10^{6}$ promastigotes $/ \mathrm{mL}$ in M199. After an incubation for $24 \mathrm{~h}$ at $28{ }^{\circ} \mathrm{C}$, the cell culture density was determined using a cell counter (CASY model TT, Roche Diagnostics) with a $60 \mu \mathrm{m}$ capillary and exclusion of particles with a pseudo diameter below $2.0 \mu \mathrm{m}$. The cell density was adjusted again to $1 \times 10^{6}$ promastigotes $/ \mathrm{mL}$ in a new flask. This procedure was repeated for $4 \mathrm{~d}$. The doubling time (DT) was calculated using the following formula.

$$
\mathrm{DT}=\frac{24}{\log _{2}\left(\frac{\text { parasite number after } 24 \mathrm{~h}}{\text { parasite initial number }}\right)}
$$

Susceptibility Assays. The susceptibility to MF was determined by an MTT assay. ${ }^{54}$ Briefly, $2 \times 10^{6}$ ( $\mathrm{L}$. braziliensis) or $2 \times 10^{5}$ (L. major) log-phase promastigotes were incubated in the presence of increasing concentrations of MF for $24 \mathrm{~h}$ (SR clones) or $48 \mathrm{~h}$ (knockout mutants). MF concentrations employed for L. braziliensis were 400, 280, 240, $200,140,120,100,70,60,50$, and $35 \mu \mathrm{M}$, and for L. major they were $120,90,80,70,60,45,30,15,7.5,3.75$, and 1.875 $\mu \mathrm{M}$. The cell viability was then determined by an incubation with $5 \mathrm{mg} / \mathrm{mL}$ MTT followed by cell lysis with 4\% SDS and optical density (OD) measurement at 690 and $595 \mathrm{~nm}$. OD values were then converted in $\mathrm{EC}_{50}$ values by sigmoidal regression curves using GraphPad Prism 6 software. Susceptibility assays were conducted in triplicate, and at least three independent experiments were performed.

Uptake of MT-EtBDPY. The uptake of MT-EtBDPY was evaluated as described in Espada et al. ${ }^{21}$ Briefly, log-phase Leishmania promastigotes were incubated in HEPES- $\mathrm{NaCl}$ buffer (21 mM HEPES, $137 \mathrm{mM} \mathrm{NaCl}, 5 \mathrm{mM} \mathrm{KCl}, 0.7 \mathrm{mM}$ $\mathrm{NaH}_{2} \mathrm{PO}_{4}, 6 \mathrm{mM}$ glucose, $\left.\mathrm{pH} 7.05\right)$ supplemented with $0.3 \%$ $(\mathrm{w} / \mathrm{v})$ bovine serum albumin (BSA) and $500 \mu \mathrm{M}$ phenylmethylsulfonyl fluoride (PMSF) (Sigma-Aldrich) for $15 \mathrm{~min}$ at $28{ }^{\circ} \mathrm{C}$. After this period, $1 \mu \mathrm{M}$ MT-EtBDP was added, and the incubation was continued for $5 \mathrm{~min}$ at $28{ }^{\circ} \mathrm{C}$. Parasites were washed three times with $\mathrm{HEPES}-\mathrm{NaCl}$ containing $0.3 \% \mathrm{BSA}$ to remove the noninternalized labeled molecules. The parasites were then suspended in PBS, and the fluorescence intensity was measured using a BD Accuri C6 flow cytometer (BD Biosciences). Statistical analyses were performed using oneway ANOVA followed by Tukey's multiple comparison tests using Graph Pad Prism 6. Values are reported with the standard error of measure (SEM).

Fluorescence Microscopy. For MT-EtBDP uptake analysis, after noninternalized molecules were washed, a fraction of the parasites was incubated in PBS with $10 \mu \mathrm{g} /$ $\mathrm{mL}$ Hoechst 33342. Parasites were pelleted, suspended in PBS, and then placed on a microscope slide inside a small area marked with a liquid blocker pen. A coverslip was applied, and the living cells were immediately imaged in a Zeiss Axioimager.Z2 microscope with a $63 \times$ numerical aperture (NA) 1.40 oil immersion objective and a Hamamatsu ORCAFlash4.0 camera. The filters used for Hoechst 33342 and MTEtBDP were $350 / 461 \mathrm{~nm}$ (excitation/emission) and 527/536 $\mathrm{nm}$, respectively. As a background control, WT Cas9/T7 untagged and/or that did not receive the ligands or fluorescent molecules were imaged using the specific filters at the same exposure time ( 0.04 and $4 \mathrm{~s})$. Images were processed using Fiji. $^{55}$

\section{ASSOCIATED CONTENT}

\section{SI Supporting Information}

The Supporting Information is available free of charge at https://pubs.acs.org/doi/10.1021/acsinfecdis.0c00857. 
- PCR confirmation of SR cassette for ros 3 overexpression in L. braziliensis $\mathrm{T} 2$ and L. major FV-1 clones (PDF)

- List of all oligonucleotides used in this work (PDF)

\section{AUTHOR INFORMATION}

\section{Corresponding Author}

Caroline R. Espada - Departamento de Parasitologia, Instituto de Ciencias Biomédicas, Universidade de São Paulo, São Paulo, Brazil; † orcid.org/0000-0003-1391-8091; Phone: +55-16-33153053; Email: caroline.respada@ usp.br

\section{Authors}

Andreia Albuquerque-Wendt - Sir William Dunn School of Pathology, University of Oxford, Oxford, United Kingdom; Global Health and Tropical Medicine, Instituto de Higiene e Medicina Tropical, Universidade de Lisboa, Lisboa, Portugal; Wellcome Centre for Integrative Parasitology, Institute of Infection, Immunity \& Inflammation, College of Medical Veterinary and Life Sciences, University of Glasgow, Glasgow, United Kingdom

Valentín Hornillos - Departamento de Química Orgánica, Universidad de Sevilla and Centro de Innovación en Química Avanzada, Sevilla, Spain; 다이.org/0000-0002-61819146

Eva Gluenz - Sir William Dunn School of Pathology, University of Oxford, Oxford, United Kingdom; Wellcome Centre for Integrative Parasitology, Institute of Infection, Immunity \& Inflammation, College of Medical Veterinary and Life Sciences, University of Glasgow, Glasgow, United Kingdom

Adriano C. Coelho - Departamento de Biologia Animal, Instituto de Biologia, Universidade Estadual de Campinas, Campinas, Brazil; $\odot$ orcid.org/0000-0002-0659-6269

Silvia R. B. Uliana - Departamento de Parasitologia, Instituto de Cîncias Biomédicas, Universidade de São Paulo, São Paulo, Brazil

Complete contact information is available at:

https://pubs.acs.org/10.1021/acsinfecdis.0c00857

\section{Author Contributions}

C.R.E.: Conceptualization, investigation, formal analysis, methodology, visualization, writing, reviewing and editing. A.A.-W.: Conceptualization, methodology, formal analysis, reviewing, and editing. V.H.: development of MT-EtBDP and reviewing. E.G.: Conceptualization, methodology, formal analysis, reviewing, and editing. A.C.C.: Conceptualization, methodology, writing, reviewing, and editing. S.R.B.U.: Conceptualization, methodology, formal analysis, writing, reviewing, and editing.

Notes

The authors declare no competing financial interest.

\section{ACKNOWLEDGMENTS}

We want to thank J. K. U. Yokoyama-Yasunaka and H. Banks (supported by Wellcome Trust Grant [104627/Z/14/Z] to K. Gull) for the valuable lab support. This work was supported by Fundação de Amparo à Pesquisa do Estado de São Paulo (FAPESP 2015/09080-2, 2016/23405-4, 2016/21171-6 and 2018/25299-2). S.R.B.U. is the recipient of a senior researcher scholarship from CNPq. A.C.C. has also, in part, received funding from UK Research and Innovation via the Global
Challenges Research Fund under the grant "A Global Network for Neglected Tropical Diseases” (Grant No. MR/P027989/ 1). V.H. acknowledges the predoctoral Grant FPI (2004-2009) from Ministerio de Educación y Ciencia of Spain (BQU200304413) and the Contract RYC-2017-22294 from the Spanish Ministerio de Ciencia e Innovación. A.A.-W. is the recipient of a Marie Sklodowska-Curie Individual Fellowship (transLEISHion-EU FP7, No. 798736) and acknowledges Fundação para a Ciência e a Tecnologia for funds to GHTM (UID/ 04413/2020). E.G. is a Royal Society University Research Fellow and supported through the WCIP core Wellcome Centre Award No. 104111/Z/14/Z.

\section{REFERENCES}

(1) Grimaldi, G., Jr., Tesh, R. B., and McMahon-Pratt, D. (1989) A review of the geographic distribution and epidemiology of leishmaniasis in the New World. Am. J. Trop. Med. Hyg. 41 (6), 687-725.

(2) Silveira, F. T. (2019) What makes mucosal and anergic diffuse cutaneous leishmaniases so clinically and immunopathogically different? A review in Brazil. Trans. R. Soc. Trop. Med. Hyg. 113, 505.

(3) Cincurá, C., de Lima, C. M. F., Machado, P. R. L., Oliveira-Filho, J., Glesby, M. J., Lessa, M. M., and Carvalho, E. M. (2017) Mucosal leishmaniasis: A Retrospective Study of 327 Cases from an Endemic Area of Leishmania (Viannia) braziliensis. Am. J. Trop. Med. Hyg. 97 (3), 761-766.

(4) Burza, S., Croft, S. L., and Boelaert, M. (2018) Leishmaniasis. Lancet 392 (10151), 951-970.

(5) Uliana, S. R. B., Trinconi, C. T., and Coelho, A. C. (2018) Chemotherapy of leishmaniasis: present challenges. Parasitology 145 (4), 464-480.

(6) Machado, P. R., Ampuero, J., Guimaraes, L. H., Villasboas, L., Rocha, A. T., Schriefer, A., Sousa, R. S., Talhari, A., Penna, G., and Carvalho, E. M. Miltefosine in the treatment of cutaneous leishmaniasis caused by Leishmania braziliensis in Brazil: a randomized and controlled trial. PLoS neglected tropical diseases 2010, 4 (12). DOI: 10.1371 /journal.pntd.0000912.

(7) Machado, P. R. L., Prates, F. V. O., Boaventura, V., Lago, T., Guimarães, L. H., Schriefer, A., Corte, T. W. F., Penna, G., Barral, A., Barral-Netto, M., and Carvalho, E. M. (2020) A double-bind and randomized trial to evaluate Miltefosine and topical GM-CSF in the treatment of cutaneous leishmaniasis caused by Leishmania braziliensis in Brazil. Clin. Infect. Dis., DOI: 10.1093/cid/ciaa1337.

(8) Sunyoto, T., Potet, J., and Boelaert, M. Why miltefosine-a lifesaving drug for leishmaniasis-is unavailable to people who need it the most. BMJ. global health 2018, 3 (3). DOI: 10.1136/bmjgh-2018000709.

(9) Brasil, Ministério da Saúde. Secretaria de Ciência, Tecnologia e Insumos Estratégicos. Comissão Nacional de Inocorporação de Tecnologia no SUS Miltefosina para o tratamento da Leishmaniose Tegumentar. http://conitec.gov.br/images/Relatorios/2018/ Relatorio_Miltefosina_LeishmanioseTegumentar.pdf (accessed 2021-03-14)

(10) Chrusciak-Talhari, A., Dietze, R., Chrusciak Talhari, C., da Silva, R. M., Gadelha Yamashita, E. P., de Oliveira Penna, G., Lima Machado, P. R., and Talhari, S. (2011) Randomized controlled clinical trial to access efficacy and safety of miltefosine in the treatment of cutaneous leishmaniasis Caused by Leishmania (Viannia) guyanensis in Manaus, Brazil. Am. J. Trop. Med. Hyg. 84 (2), 255-260.

(11) Dorlo, T. P., Balasegaram, M., Beijnen, J. H., and de Vries, P. J. (2012) Miltefosine: a review of its pharmacology and therapeutic efficacy in the treatment of leishmaniasis. J. Antimicrob. Chemother. 67 (11), 2576-2597.

(12) Sundar, S., Singh, A., Rai, M., Prajapati, V. K., Singh, A. K. Ostyn, B., Boelaert, M., Dujardin, J. C., and Chakravarty, J. (2012) Efficacy of miltefosine in the treatment of visceral leishmaniasis in India after a decade of use. Clin. Infect. Dis. 55 (4), 543-550. 
(13) Srivastava, S., Mishra, J., Gupta, A. K., Singh, A., Shankar, P., and Singh, S. Laboratory confirmed miltefosine resistant cases of visceral leishmaniasis from India. Parasites \& Vectors 2017, 10 (1). DOI: 10.1186/s13071-017-1969-z.

(14) Rijal, S., Ostyn, B., Uranw, S., Rai, K., Bhattarai, N. R., Dorlo, T. P., Beijnen, J. H., Vanaerschot, M., Decuypere, S., Dhakal, S. S., Das, M. L., Karki, P., Singh, R., Boelaert, M., and Dujardin, J. C. (2013) Increasing failure of miltefosine in the treatment of kala-azar in Nepal and the potential role of parasite drug resistance, reinfection, or noncompliance. Clin. Infect. Dis. 56 (11), 1530-1538.

(15) Mondelaers, A., Sanchez-Canete, M. P., Hendrickx, S., Eberhardt, E., Garcia-Hernandez, R., Lachaud, L., Cotton, J., Sanders, M., Cuypers, B., Imamura, H., Dujardin, J. C., Delputte, P., Cos, P., Caljon, G., Gamarro, F., Castanys, S., and Maes, L. Genomic and Molecular Characterization of Miltefosine Resistance in Leishmania infantum Strains with Either Natural or Acquired Resistance through Experimental Selection of Intracellular Amastigotes. PloS one 2016, 11 (4). DOI: 10.1371/journal.pone.0154101.

(16) Cojean, S., Houze, S., Haouchine, D., Huteau, F., Lariven, S., Hubert, V., Michard, F., Bories, C., Pratlong, F., Le Bras, J., Loiseau, P. M., and Matheron, S. (2012) Leishmania resistance to miltefosine associated with genetic marker. Emerging Infect. Dis. 18 (4), 704-706.

(17) Carnielli, J. B. T., Monti-Rocha, R., Costa, D. L., Molina Sesana, A., Pansini, L. N. N., Segatto, M., Mottram, J. C., Costa, C. H. N., Carvalho, S. F. G., and Dietze, R. (2019) Natural Resistance of Leishmania infantum to Miltefosine Contributes to the Low Efficacy in the Treatment of Visceral Leishmaniasis in Brazil. Am. J. Trop. Med. Hyg. 101 (4), 789-794.

(18) Perez-Victoria, F. J., Gamarro, F., Ouellette, M., and Castanys, S. (2003) Functional cloning of the miltefosine transporter. A novel P-type phospholipid translocase from Leishmania involved in drug resistance. J. Biol. Chem. 278 (50), 49965-49971.

(19) Perez-Victoria, F. J., Castanys, S., and Gamarro, F. (2003) Leishmania donovani resistance to miltefosine involves a defective inward translocation of the drug. Antimicrob. Agents Chemother. 47 (8), 2397-2403.

(20) Coelho, A. C., Trinconi, C. T., Costa, C. H., and Uliana, S. R. In Vitro and In Vivo Miltefosine Susceptibility of a Leishmania amazonensis Isolate from a Patient with Diffuse Cutaneous Leishmaniasis. PLoS Negl Trop Dis 2014, 8 (7). DOI: 10.1371/ journal.pntd.0002999.

(21) Espada, C. R., Magalhaes, R. M., Cruz, M. C., Machado, P. R., Schriefer, A., Carvalho, E. M., Hornillos, V., Alves, J. M., Cruz, A. K., Coelho, A. C., and Uliana, S. R. B. (2019) Investigation of the pathways related to intrinsic miltefosine tolerance in Leishmania (Viannia) braziliensis clinical isolates reveals differences in drug uptake. Int. J. Parasitol.: Drugs Drug Resist. 11, 139.

(22) Sanchez-Canete, M. P., Carvalho, L., Perez-Victoria, F. J., Gamarro, F., and Castanys, S. (2009) Low plasma membrane expression of the miltefosine transport complex renders Leishmania braziliensis refractory to the drug. Antimicrob. Agents Chemother. 53 (4), 1305-1313.

(23) García-Sánchez, S., Sánchez-Cañete, M. P., Gamarro, F., and Castanys, S. (2014) Functional role of evolutionarily highly conserved residues, $\mathrm{N}$-glycosylation level and domains of the Leishmania miltefosine transporter-Cdc50 subunit. Biochem. J. 459 (1), 83-94.

(24) Perez-Victoria, F. J., Sanchez-Canete, M. P., Castanys, S., and Gamarro, F. (2006) Phospholipid translocation and miltefosine potency require both $L$. donovani miltefosine transporter and the new protein LdRos3 in Leishmania parasites. J. Biol. Chem. 281 (33), 23766-23775.

(25) Hanson, P. K., Malone, L., Birchmore, J. L., and Nichols, J. W. (2003) Lem $3 p$ is essential for the uptake and potency of alkylphosphocholine drugs, edelfosine and miltefosine. J. Biol. Chem. 278 (38), 36041-36050.

(26) Espada, C. R., Ribeiro-Dias, F., Dorta, M. L., Pereira, L. I. A., Carvalho, E. M., Machado, P. R., Schriefer, A., Yokoyama-Yasunaka, J. K. U., Coelho, A. C., and Uliana, S. R. B. (2017) Susceptibility to
Miltefosine in Brazilian Clinical Isolates of Leishmania (Viannia) braziliensis. Am. J. Trop Med. Hyg 96 (3), 656-659.

(27) Downing, T., Imamura, H., Decuypere, S., Clark, T. G., Coombs, G. H., Cotton, J. A., Hilley, J. D., de Doncker, S., Maes, I., Mottram, J. C., Quail, M. A., Rijal, S., Sanders, M., Schonian, G., Stark, O., Sundar, S., Vanaerschot, M., Hertz-Fowler, C., Dujardin, J. C., and Berriman, M. (2011) Whole genome sequencing of multiple Leishmania donovani clinical isolates provides insights into population structure and mechanisms of drug resistance. Genome Res. 21 (12), $2143-2156$

(28) Leprohon, P., Legare, D., Raymond, F., Madore, E., Hardiman, G., Corbeil, J., and Ouellette, M. (2009) Gene expression modulation is associated with gene amplification, supernumerary chromosomes and chromosome loss in antimony-resistant Leishmania infantum. Nucleic Acids Res. 37 (5), 1387-1399.

(29) Cohen-Freue, G., Holzer, T. R., Forney, J. D., and McMaster, W. R. (2007) Global gene expression in Leishmania. Int. J. Parasitol. 37 (10), 1077-1086.

(30) Clayton, C. E. (2016) Gene expression in Kinetoplastids. Curr. Opin. Microbiol. 32, 46-51.

(31) Cupolillo, E., Brahim, L. R., Toaldo, C. B., De Oliveira-Neto, M. P., De Brito, M. E., Falqueto, A., De Farias Naiff, M., and Grimaldi, G., Jr. (2003) Genetic Polymorphism and Molecular Epidemiology of Leishmania (Viannia) braziliensis from Different Hosts and Geographic Areas in Brazil. J. Clin Microbiol 41 (7), 3126-3132.

(32) Kebede, A., De Doncker, S., Arevalo, J., Le Ray, D., and Dujardin, J. C. (1999) Size-polymorphism of mini-exon gene-bearing chromosomes among natural populations of Leishmania, subgenus Viannia. Int. J. Parasitol. 29 (4), 549-557.

(33) Laffitte, M. N., Leprohon, P., Papadopoulou, B., and Ouellette, M. Plasticity of the Leishmania genome leading to gene copy number variations and drug resistance. F1000Research 2016, 5. DOI: 10.12688/f1000research.9218.1.

(34) Gazdag, E. M., Cirstea, I. C., Breitling, R., Lukes, J., Blankenfeldt, W., and Alexandrov, K. (2010) Purification and crystallization of human $\mathrm{Cu} / \mathrm{Zn}$ superoxide dismutase recombinantly produced in the protozoan Leishmania tarentolae. Acta Crystallogr. Sect. F: Struct. Biol. Cryst. Commun. 66, 871-877.

(35) Bastos, M. S. e, Souza, L. A. d., Onofre, T. S., Silva Junior, A., Almeida, M. R. d., Bressan, G. C., and Fietto, J. L. R. (2017) Achievement of constitutive fluorescent pLEXSY-egfp Leishmania braziliensis and its application as an alternative method for drug screening in vitro. Memorias do Instituto Oswaldo Cruz 112 (2), 155159.

(36) Rezaei, Z., Van Reet, N., Pouladfar, G., Kühne, V., Ramezani, A., Sarkari, B., Pourabbas, B., and Büscher, P. Expression of a rK39 homologue from an Iranian Leishmania infantum isolate in Leishmania tarentolae for serodiagnosis of visceral leishmaniasis. Parasites \& vectors 2019, 12 (1). DOI: 10.1186/s13071-019-3839-3.

(37) de Souza, L. M. B., Carvalho, J., Bates, M. D., Petterle, R. R. Thomaz-Soccol, V., and Bates, P. A. Production of a kinesin-related recombinant protein (Lbk39) from Leishmania braziliensis by Leishmania tarentolae promastigotes and its application in the serodiagnosis of leishmaniasis. One health (Amsterdam, Netherlands) 2019, 8. DOI: 10.1016/j.onehlt.2019.100111.

(38) Freitas Castro, F., Ruy, P. C., Nogueira Zeviani, K., Freitas Santos, R., Simoes Toledo, J., and Kaysel Cruz, A. (2017) Evidence of putative non-coding RNAs from Leishmania untranslated regions. Mol. Biochem. Parasitol. 214, 69-74.

(39) Fernandes, K. S., de Souza, P. E., Dorta, M. L., and Alonso, A. (2017) The cytotoxic activity of miltefosine against Leishmania and macrophages is associated with dynamic changes in plasma membrane proteins. Biochimica et biophysica acta. Biochim. Biophys. Acta, Biomembr. 1859 (1), 1-9.

(40) Coelho, A. C., Leprohon, P., and Ouellette, M. Generation of Leishmania hybrids by whole genomic DNA transformation. PLoS Negl Trop Dis 2012, 6 (9). DOI: 10.1371/journal.pntd.0001817.

(41) Shaw, C. D., Lonchamp, J., Downing, T., Imamura, H., Freeman, T. M., Cotton, J. A., Sanders, M., Blackburn, G., Dujardin, J. 
C., Rijal, S., Khanal, B., Illingworth, C. J., Coombs, G. H., and Carter, K. C. (2016) In vitro selection of miltefosine resistance in promastigotes of Leishmania donovani from Nepal: genomic and metabolomic characterization. Mol. Microbiol. 99 (6), 1134-1148.

(42) Sundar, S., Jha, T. K., Thakur, C. P., Engel, J., Sindermann, H., Fischer, C., Junge, K., Bryceson, A., and Berman, J. (2002) Oral miltefosine for Indian visceral leishmaniasis. N. Engl. J. Med. 347 (22), $1739-1746$

(43) Jha, T. K., Sundar, S., Thakur, C. P., Bachmann, P., Karbwang, J., Fischer, C., Voss, A., and Berman, J. (1999) Miltefosine, an oral agent, for the treatment of Indian visceral leishmaniasis. N. Engl. J. Med. 341 (24), 1795-1800.

(44) Castro, M. D., Gomez, M. A., Kip, A. E., Cossio, A., Ortiz, E., Navas, A., Dorlo, T. P., and Saravia, N. G. Pharmacokinetics of Miltefosine in Children and Adults with Cutaneous Leishmaniasis. Antimicrob. Agents Chemother. 2017, 61 (3). DOI: 10.1128/ aac.02198-16.

(45) Dorlo, T. P., van Thiel, P. P., Huitema, A. D., Keizer, R. J., de Vries, H. J., Beijnen, J. H., and de Vries, P. J. (2008) Pharmacokinetics of miltefosine in Old World cutaneous leishmaniasis patients. Antimicrob. Agents Chemother. 52 (8), 2855-2860.

(46) Hornillos, V., Carrillo, E., Rivas, L., Amat-Guerri, F., and Acuna, A. U. (2008) Synthesis of BODIPY-labeled alkylphosphocholines with leishmanicidal activity, as fluorescent analogues of miltefosine. Bioorg. Med. Chem. Lett. 18 (24), 6336-6339.

(47) Beneke, T., Madden, R., Makin, L., Valli, J., Sunter, J., and Gluenz, E. (2017) A CRISPR Cas9 high-throughput genome editing toolkit for kinetoplastids. Royal Society open science 4, 1-16.

(48) Niimi, T. (2012) Recombinant protein production in the eukaryotic protozoan parasite Leishmania tarentolae: a review. Methods Mol. Biol. (N. Y., NY, U. S.) 824, 307-315.

(49) Coburn, C. M., Otteman, K. M., McNeely, T., Turco, S. J., and Beverley, S. M. (1991) Stable DNA transfection of a wide range of trypanosomatids. Mol. Biochem. Parasitol. 46 (1), 169-179.

(50) Beverley, S. M., and Coburn, C. M. (1990) Recurrent de novo appearance of small linear DNAs in Leishmania major and relationship to extra-chromosomal DNAs in other species. Mol. Biochem. Parasitol. 42 (1), 133-141.

(51) Rotureau, B., Gego, A., and Carme, B. (2005) Trypanosomatid protozoa: a simplified DNA isolation procedure. Exp. Parasitol. 111 (3), 207-209.

(52) Livak, K. J., and Schmittgen, T. D. (2001) Analysis of relative gene expression data using real-time quantitative PCR and the 2(Delta Delta $\mathrm{C}(\mathrm{T})$ ) Method. Methods (Amsterdam, Neth.) 25 (4), 402-408.

(53) Beneke, T., and Gluenz, E. (2019) LeishGEdit: A Method for Rapid Gene Knockout and Tagging Using CRISPR-Cas9. Methods Mol. Biol. (N. Y., NY, U. S.) 1971, 189-210.

(54) Zauli-Nascimento, R. C., Miguel, D. C., Yokoyama-Yasunaka, J. K., Pereira, L. I., Pelli de Oliveira, M. A., Ribeiro-Dias, F., Dorta, M. L., and Uliana, S. R. (2010) In vitro sensitivity of Leishmania (Viannia) braziliensis and Leishmania (Leishmania) amazonensis Brazilian isolates to meglumine antimoniate and amphotericin B. Tropical Medicine \& International Health 15 (1), 68-76.

(55) Schindelin, J., Arganda-Carreras, I., Frise, E., Kaynig, V., Longair, M., Pietzsch, T., Preibisch, S., Rueden, C., Saalfeld, S., Schmid, B., Tinevez, J. Y., White, D. J., Hartenstein, V., Eliceiri, K., Tomancak, P., and Cardona, A. (2012) Fiji: an open-source platform for biological-image analysis. Nat. Methods 9 (7), 676-682. 